> Köhler et Milstein ont publié en 1975 une méthode de production d'anticorps monoclonaux de souris aux spécificités prédéfinies, travail qui fut récompensé par le prix Nobel en 1984. Depuis, le domaine s'est développé rapidement, de nouvelles méthodes de production ont été créées : technologie de l'ADN recombinant, technique de phage display, création d'animaux génétiquement modifiés. Après la première application clinique des anticorps monoclonaux d'origine murine, de nouvelles classes d'anticorps monoclonaux chimériques, humanisés et humains sont apparues. Ces anticorps associent une meilleure efficacité et moins d'effets indésirables. Les développements menés depuis une trentaine d'années ont abouti à la production de plus de vingt-cinq anticorps à visée thérapeutique disposant d'une autorisation de mise sur le marché pour diverses applications, par exemple dans le domaine de l'oncologie, des maladies inflammatoires, des maladies cardiaques et de la transplantation. Des médicaments pour de nombreuses autres applications sont en cours de développement. <

\section{Conséquences réglementaires de I'utilisation des anticorps monoclonaux}

Friedrich Lackner, Marie-Emmanuelle Behr-Gross

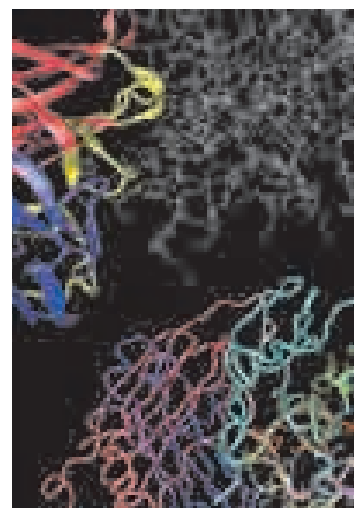

F. Lackner : AGES Pharmmed, Austrian Agency for Health and Food Safety, OMCL, Possingergasse 38 , A-1160 Vienne, Autriche. friedrich.lackner@ages.at M.E. Behr-Gross : EDQM, Strasbourg, France.

la production de l'anticorps (désignées sous le terme de «substrats cellulaires») doivent être stables et les anticorps obtenus par génie génétique («anticorps recombinants ») doivent être conformes au chapitre des recommandations de l'EMEA sur «Production et contrôle de la qualité des médicaments dérivés de la technologie de l'ADN recombinant $(3 \mathrm{Abl} \mathrm{A})$ » et aux directives d'harmonisation internationale $\mathrm{ICH}^{1}$ $\mathrm{Q} 5 \mathrm{~A}$ (sécurité virale), $\mathrm{Q} 5 \mathrm{~B}$ (expression des constructions) et $\mathrm{Q} 5 \mathrm{D}$ (substrats cellulaires). L'immortalisation de lymphocytes $B$ par transformation ou fusion cellulaire peut être nécessaire, mais cette approche doit être considérée avec prudence en ce qui concerne la sécurité (virale ou liée aux TSE²) et l'efficacité.

La monographie de la Pharmacopée européenne (Ph. eur.), Anticorps monoclonaux pour usage humain (2031), est actuellement en cours de révision et un nouveau texte a été publié pour consultation dans le forum de la $\mathrm{Ph}$. eur., Pharmeuropa $^{3}$ (PA/PH/Exp. MAB/T(08)2 ANP) [2]. Ce projet

${ }^{1} \mathrm{ICH}$ : International conference on harmonisation of technical requirements for the registration of pharmaceuticals for human use. L'objectif est d'établir un forum de discussion entre les autorités de régulation et l'industrie pharmaceutique sur les différences (réelles et perçues) existant entre les États-Unis, l'Europe et le Japon en ce qui concerne les impératifs techniques requis pour l'enregistrement de produits pharmaceutiques. Ce forum doit favoriser et accélérer l'accessibilité de nouveaux médicaments aux patients. ${ }^{2}$ TSE : transmissible spongiform encephalopathies.

3 «Le forum des lecteurs: toute l'actualité de la Pharmacopée européenne, en 4 numéros par an. Chaque numéro publie : les enquêtes publiques sur des projets de textes européens ou sur des questions de politique générale, les dernières annonces officielles sur les monographies nouvellement adoptées par la Commission européenne de Pharmacopée, l'actualité de l'harmonisation internationale. Pharmeuropa est disponible en français et en anglais, au numéro ou par abonnement, en version imprimée et internet. » (reproduit de http://www.edqm.eu/fr) 


\begin{tabular}{|c|c|c|}
\hline & $\begin{array}{l}\text { Directive de l'EMEA } \\
\text { EMEA/CHMP/BWP/157653/2007 } \\
(*)\end{array}$ & $\begin{array}{l}\text { Projet de monographie de la Ph. eur } \\
\text { PA/PH/Exp. MAB/T (08) } 2 \text { ANP } \\
(* *)\end{array}$ \\
\hline Base juridique & $\begin{array}{l}\text { Annexe I de la directive } 2001 / 83 / C \varepsilon \text { telle } \\
\text { qu'amendée par la directive } 2003 / 83 / C \varepsilon\end{array}$ & $\begin{array}{l}\text { Directive } 2001 / 83 / \varepsilon C \text { telle qu'amendée par la } \\
\text { directive } 2003 / 83 / \varepsilon C\end{array}$ \\
\hline Développement & $\begin{array}{l}\text { 4.1. Justification des propriétés immunochimi- } \\
\text { ques, fonctions effectrices, épitopes potentielle- } \\
\text { ment immunogènes, substrat cellulaire. }\end{array}$ & - \\
\hline Production & $\begin{array}{l}\text { 4.2.2. Plateforme de production } \\
\text { 4.2.3. Sécurité virale et TSE }\end{array}$ & $\begin{array}{l}\text { - Validation de la méthode de production } \\
\text { - Validation du procédé, caractérisation du pro- } \\
\text { duit, intermédiaires de production, titrage biologi- } \\
\text { que, préparation de référence, définition du lot } \\
\text { - Cellule source, lignée cellulaire productrice } \\
\text { de l'anticorps monoclonal, banques de cellules, } \\
\text { culture et récolte, purification }\end{array}$ \\
\hline Caractérisation & $\begin{array}{l}\text { 4.3.1-4.3.3. Propriétés physicochimiques, immu- } \\
\text { nologiques, activité biologique }\end{array}$ & - Caractères, identification, tests, essai \\
\hline Identité, pureté, spécifications & 4.3.4. Pureté et contamination & - Identité, pureté, impuretés du vrac final et lot final \\
\hline Stockage et étiquetage & - & -Conservation, date de péremption, étiquetage \\
\hline
\end{tabular}

Tableau I. Cadre réglementaire (vue d'ensemble). Dans ce tableau, certains aspects clés des deux plus importants documents de réglementation sont comparés. Comme ces documents diffèrent par leur structure, seule une vue d'ensemble est donnée sans comparer les textes en détail.

* La nouvelle directive EMEA/CHMP/BWP/157653/2007 de l'Agence européenne du médicament (EMEA) sur «le développement, la fabrication, la caractérisation et les spécifications pour les anticorps monoclonaux et les produits similaires » remplace l'ancienne sur «la production et le

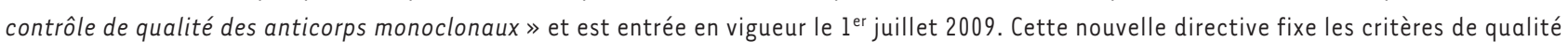
pour les anticorps monoclonaux soumis à une autorisation de mise sur le marché et destinés à des usages thérapeutiques, prophylactiques et de diagnostic in vivo. Cette directive s'applique également aux anticorps monoclonaux utilisés comme réactifs et aux produits connexes tels que les fragments conjugués (pour radiomarquage par exemple) et les protéines de fusion.

** Le projet de monographie Anticorps monoclonaux pour usage humain de la Pharmacopée européenne (Ph. eur.) (PA/PH/Exp. MAB/T (08) 2 ANP) a été publié pour consultation en janvier 2009. La monographie établit les règles et spécifications à suivre au cours de la production, des essais et du stockage des anticorps monoclonaux. Elle s'applique également aux anticorps monoclonaux thérapeutiques et prophylactiques et aux anticorps utilisés pour le diagnostic in vivo. Sont exclus du champ de cette monographie les anticorps polyclonaux, les anticorps utilisés comme réactifs pour la fabrication de médicaments et les anticorps monoclonaux produits par la technique dite de l'ascite.

de révision comporte une section qui définit les types d’Acm obtenus par les techniques d'ingénierie actuellement disponibles reposant sur la technique de l'ADN recombinant ( $A D N r$ ) ainsi que le champ d'application de la monographie. Les Acm obtenus à partir de lignées de cellules modifiées par recombinaison de l'ADN doivent se conformer aux exigences de la monographie de la Ph. eur. Produits de technologie de l'ADN recombinant (0784). La monographie Anticorps monoclonaux pour usage humain s'applique aux Acm thérapeutiques et prophylactiques et à usage de diagnostic in vivo, mais pas aux Acm utilisés comme principe actif dans la fabrication de médicaments. Les exigences requises pour la fabrication d'Acm chez la souris (par induction d'ascite) sont définies par l'autorité compétente nationale concernée et ne sont pas couvertes par cette monographie. Compte tenu des risques de contamination par des agents étrangers, de la réglementation sur la protection des animaux et de l'existence de systèmes de production alternatifs, ce mode de production n'est plus utilisé pour les Acm qui sont développés aujourd'hui.

\section{Cadre réglementaire pour la production d'anticorps monoclonaux}

\section{Développement, fabrication, caractérisation et spécifications des anticorps monoclonaux et produits similaires ${ }^{4}$}

La plateforme de fabrication d'Acm a été un point de discussion important, principalement au cours de

${ }^{4}$ EMEA/CHMP/BWP/157653/2007. CHMP : Committee for medicinal products for human use. 
l'élaboration des recommandations de I'EMEA (voir 4.2.2.). Le terme plateforme désigne une stratégie de production utilisée par des fabricants experts fondée sur des procédés de fabrication similaires incluant des cultures cellulaires et des étapes de purification prédéfinies $(\rightarrow)$. Les données et conclusions tirées d'expériences préalables de production, si elles sont justifiées $(\rightarrow)$ voir $\mathbf{0}$. Cochet et M. Chartrain, page 1078 ; S. Olivier et M. Mehtali, page 1163 et documentées, peuvent être utilisées pour corroborer ou remplacer partiellement les données habituellement requises pour l'évaluation du processus commercial à présenter.

Les questions de sécurité virale et du risque de transmission de l'agent de l'encéphalopathie spongiforme transmissible (TSE) (voir 4.2.3) sont posées et la validation de l'inactivation virale dans la ligne de production selon la directive $25 \mathrm{~A}$ de I'ICH est soulignée. Si des effets spécifiques du produit sur la capacité de réduction du virus ne peuvent pas être exclus, des procédures de confirmation utilisant des produits intermédiaires spécifiques pour le processus doivent être mises en œuvre. La note d'orientation (EMEA/410/01): Minimiser les risques de transmission des agents de l'encéphalopathie spongiforme animale par médicaments humains et vétérinaires doit être prise en compte si des matériaux d'origine bovine ont été utilisés au cours du développement ou de la fabrication.

\section{Monographie de la Pharmacopée européenne:}

\section{anticorps monoclonaux pour usage humain (2031)}

Les critères de validation du processus de production sont détaillés dans le projet de monographie de la Ph. eur. Ils concernent la cohérence de la production, l'élimination des agents infectieux, la suppression des impuretés liées au processus de production ou associées au produit, la spécificité et l'activité spécifique de l'anticorps, l'absence de substances pyrogènes autres que les endotoxines et la réutilisation des composants de purification. Des paragraphes spéciaux détaillent les exigences propres à l'origine des cellules productrices des anticorps parentaux, aux lignées de cellules pour la production des Acm, aux banques de cellules, à la culture et à la récolte, à la purification de la substance active (expression qui remplace celle d'«anticorps monoclonaux purifiés » utilisée dans le texte original). Le test de stérilité a été remplacé par un contrôle de la charge microbienne en cours de procédé.

\section{Cadre réglementaire pour la caractérisation et les spécifications des anticorps monoclonaux}

La directive EMEA/CHMP/BWP/157653/2007 de l'EMEA indique que les anticorps monoclonaux doivent être largement caractérisés par des méthodes modernes physicochimiques, immunologiques et biologiques. La caractérisation concerne les éléments suivants: classes, sous-classes, composition des chaînes légères, structure primaire, présence de groupes sulphydryl libres et de ponts disulfures, composition en hydrates de carbone et structures secondaire et tertiaire. Les antigènes purifiés doivent être pleinement caractérisés pour déterminer l'affinité et l'avidité des anticorps, ainsi que la réactivité indésirable. La réactivité croisée pour les tissus humains doit elle aussi être documentée. Les fonctions effectrices, ADCC (antibody-dependent cellular cytotoxicity) et CDC (complement-dependent cytotoxicity) devraient être évaluées. L'activité biologique doit être évaluée in vitro et in vivo. La pureté et les contaminants doivent être caractérisés en utilisant une combinaison de méthodes orthogonales basées sur différentes propriétés physiques et chimiques de $(\rightarrow)$ voir L. Manache séparation $(\rightarrow)$.

et al., page 1063

L'une des plus importantes modifications post-traductionnelles des anticorps est la glycosylation, la plupart des $\mathrm{Acm}$ ayant un seul site de $\mathrm{N}$-glycosylation situé dans le domaine $\mathrm{CH}_{2}$ de la portion Fc de la chaîne lourde $(\rightarrow)$.

La glycosylation de la partie Fab

$(\rightarrow)$ voir $R$. Abès et al., page 1011 ; A. Beck et al., page 1024 peut affecter l'affinité de liaison de l'antigène, la glycosylation de la partie Fc peut affecter la structure et les fonctions effectrices des IgG. La glycosylation ne dépend pas seulement du système de production de l'IgG choisi, mais également des conditions de culture cellulaire. Elle influe directement sur l'activité biologique des Acm dont le mode d'action repose sur des fonctions effectrices. Les Acm produits dans des conditions suboptimales peuvent être anormalement glycosylés, présenter un défaut d'efficacité in vivo et une immunogénicité potentiellement dangereuse chez l'homme.

Les approches analytiques peuvent être top-down ou bottom-up, c'est-à-dire que les chaînes lourdes et légères réduites ou non réduites peuvent être séparées par chromatographie, ou que l'anticorps peut être soumis à une digestion enzymatique suivie par l'analyse en spectrométrie de masse des peptides. Dans le cas d'une lignée CHO (Chinese hamster ovary) qui produit un anticorps monoclonal recombinant, les méthodes standard d'analyse comme le SDS-PAGE ${ }^{5}$, I'IEF et la $S^{2} C^{6}$ ne sont pas suffisamment précises et sensibles pour démontrer la survenue d'un événement de traduction inattendu. Seule une combinaison de MS (MALDI-TOF-MS et ESI-MS) et RP$\mathrm{HPLC}^{7}$ des protéines de grande taille permet de mettre en évidence cet événement.

Les tests à effectuer sur le produit final sont énumérés dans la monographie de la Ph. eur. : ils doivent déterminer l'apparence, la solubilité, le pH, l'osmolalité, le volume extractible, la quantité totale de protéines, la distribution des tailles moléculaires, l'identité

${ }^{5}$ La technique du gel d'électrophorèse en conditions dénaturantes (sodium dodecyl sulfate polyacrylamide gel electrophoresis).

${ }^{6} \mathrm{IEF}$ : isoelectric focusing ; SEC : size-exclusion chromatography.

${ }^{7}$ MALDI-TOF MS : matrix-assisted laser desorption/ionization time-of-flight mass spectrometry et ESI-MS electrospray ionization mass spectrometry; RP-HPLC: reverse-phase high-performance liquid chromatography. 
moléculaire et l'intégrité structurale, la pureté, la présence de stabilisateurs, d'eau, d'endotoxines bactériennes, la stérilité. La monographie énumère aussi les tests appliqués aux anticorps modifiés. Les spécifications définies dans l'autorisation de mise sur le marché (AMM) doivent être respectées. Les prescriptions pour les essais, le stockage et l'étiquetage sont données dans la monographie.

L'apparition de particules et d'une turbidité dans les solutions peut entraîner des problèmes pour la production et la formulation des $A c m$. Les directives de l'EMEA (4.4.5, test général) indiquent que «des particules visibles et infra-visibles dans les solutions de médicaments doivent être conformes aux exigences énoncées dans la Pharmacopée européenne ». Selon les recommandations de la monographie de la Ph. eur., «les préparations liquides, reconstituées ou lyophilisées, satisfont aux limites relatives du degré d'opalescence et du degré de coloration approuvées pour le produit considéré. Elles sont sans particule visible, sauf exception justifiée et autorisée. » Dans le même temps, la Ph. eur., dans le texte intitulé Préparations parentérales (052), indique que «les solutions injectables, examinées dans des conditions appropriées de visibilité, sont limpides et pratiquement exemptes de particules ». Ces formulations démontrent que l'on doit examiner tous les problèmes des particules dans les solutions d'Acm au cas pour cas et en se fondant sur des expériences cliniques et non cliniques $(\rightarrow)$.

\section{$(\rightarrow)$ voir L. Manache et al., page 1063}

\section{Cadre réglementaire de l'enregistrement des anticorps monoclonaux}

L'utilisation des $A c m$ a été réglementée aux niveaux national et européen, en raison de l'impérieuse nécessité de limiter les risques d'effets indésirables afférents à cette nouvelle classe de médicaments. La réglementation au niveau européen est essentiellement constituée par les directives de l'Agence européenne des médicaments qui fournit des recommandations à suivre au cours du développement et de la production des Acm, et par la Pharmacopée européenne, qui fixe les normes pour garantir la qualité des produits et prescrit des tests dans ses monographies. La législation actuelle prévoit un enregistrement des Acm par l'EMEA. Même s'ils peuvent être considérés comme des produits biologiques, et à ce titre présenter une plus grande variabilité structurale par comparaison à des produits obtenus par synthèse chimique, les Acm ne sont pas inclus dans la procédure de libération des lots par les autorités de contrôle officielles (OCABR, official control authority batch release), coordonnée par la Direction européenne de la qualité du médicament et des soins de santé (DEQM). Cette procédure prévoit des tests de vérification réalisés, de manière indépendante, par les autorités sanitaires sur tous les lots, préalablement à leur commercialisation. En revanche, les Acm sont inclus dans le programme de test postmarketing des produits autorisés par la voie centralisée (CAP) pour lequel le contrôle des lots est réalisé par les autorités sanitaires à des intervalles prédéfinis après l'obtention de l'autorisation de mise sur le marché (AMM).
Cadre de réglementation pour les essais cliniques d'anticorps monoclonaux : les leçons à tirer de l'essai de l'anticorps TeGenero TGN 1412

En 2006, des complications sévères ont été observées dans l'essai clinique de phase I d'un nouvel anticorps monoclonal $(\rightarrow)$, complications dont les médias se sont largement faits l'écho. Six bénévoles ayant reçu des

$(\rightarrow)$ voir M. Pallardy, page 1130 perfusions d'anticorps TGN 1412 ont développé des complications mettant leur vie en danger, associant fièvre, douleur, état de choc, coagulation intravasculaire disséminée et dysfonctionnement multiviscéral [3]. Ce nouvel agent thérapeutique était un anticorps monoclonal humanisé de type IgG4, conçu pour être un superagoniste de la molécule de costimulation CD28 des lymphocytes T. L'administration de cet anticorps a induit chez les sujets une «tempête cytokinique», c'est-à-dire la libération rapide et massive d'une cascade de cytokines proinflammatoires. Cette toxicité n'avait pas été observée dans les études précliniques effectuées chez le macaque [4]. Cet accident a révélé les limites des données précliniques obtenues dans un modèle animal, qui, bien qu'elles soient de la plus haute importance, ne peuvent pas être fiables à $100 \%$ dans leur capacité à éliminer tout risque toxique. Par expérience, nous savons que tous les Acm ne sont pas potentiellement aussi dangereux, mais des nouveaux critères ont dû être trouvés pour définir et manier «des médicaments présentant un risque élevé ».

\section{Essais cliniques : des nouveaux critères pour les premières études chez l'homme}

La nouvelle directive EMEA/CHMP/BWP/157653/2007 comporte des exigences de qualité en ce qui concerne l'autorisation de mise sur le marché des Acm, mais ne donne aucune indication concernant les essais cliniques. Pour la compléter, et pour prendre en compte les conséquences du cas TGN1412, une nouvelle « directive sur les exigences pour les premiers essais cliniques chez l'homme des médicaments potentiellement à haut risque » (EMEA/ CHMP/SWP/28367/2007) a été élaborée par le CHMP (Committee for medicinal products for human use) de I'EMEA. Cette dernière doit s'appliquer aux nouveaux $A c m$, si les conditions suivantes sont vérifiées:

- nouveau mécanisme d'action qui interfère avec les principaux effecteurs du système immunitaire ou affecte les cytokines pléiotropes (interféron (IFN) $-\gamma$, IFN- $\alpha$ ou interleukine (IL)-10);

- cible pour laquelle on ne dispose pas de modèles animaux (épitopes ou voies métaboliques présents seulement chez l'Homme); 
- nouveau type de forme structurelle obtenu par ingénierie, par exemple Fc modifié ou anticorps divalents.

Par précaution, il a été proposé d'étendre le développement préclinique avant de démarrer les enquêtes cliniques chez l'Homme et d'inclure les sujets de façon séquentielle dans les essais de phase I chez l'Homme.

\section{Anticorps monoclonaux biosimilaires?}

Compte tenu de la forte élévation des coûts et des risques accrus qu'entraîne la mise au point d'Acm contre de nouvelles cibles, le développement de ceux qui reconnaissent des cibles bien validées et dont l'innocuité et l'efficacité ont été démontrées devient de plus en plus attrayant. Récemment, des entreprises indiennes et sud-coréennes ont développé des versions génériques (copy-cat) d’Acm autorisés pour le marché européen [5]. Certaines entreprises ont annoncé des plans d'approche des marchés de l'Union européenne et des États-Unis et I'EMEA a organisé un atelier sur ce thème à Londres le 2 juillet 2009 [6] $(\rightarrow)$. La principale motivation sous-jacente au $\quad(\rightarrow)$ voir J. Biot et développement de copies génériques d'Acm est la al.,page 1177 réduction des coûts de mise sur le marché par rapport à celui du développement de novo d'Acm, compte tenu en particulier de la longueur et du coût des essais cliniques et de la rapide expiration de brevets (vingt ans). Pour le développement d’Acm, le compétiteur réalise une sorte de «rétro-ingénierie », c'est-à-dire qu'il achète le produit original sur le marché, effectue l'analyse chimique et produit un médicament aussi similaire que possible au produit de référence, bien que les systèmes de production puissent être différents. Cela implique que les conditions de production, et donc les aspects qualitatifs, ne seront pas identiques pour les deux produits. En dépit de l'usage de méthodes d'analyse très poussées, la structure de ces macromolécules est si complexe que des différences mineures peuvent ne pas être détectées $(\rightarrow)$. Si elles existent, il faut démontrer qu'elles n'affectent ni la sécurité ni l'efficacité clinique de l'anticorps. Les modifications chimiques pouvant

$(\rightarrow)$ voir A. Beck et al., page 1024 retentir sur l'aspect clinique incluent l'introduction d'autres ponts disulfures, la désamination, l'oxydation, la cyclisation des résidus glutamines amino-terminaux, des clivages enzymatiques partiels au cours de la production, et des modifications de la glycosylation, qu'il s'agisse de l'addition différentielle de sucres ou d'autres ramifications des chaînes des sucres. En outre, des impuretés liées à des conditions de production différentes en termes de $\mathrm{pH}$, de milieu de culture cellulaire et de température contribuent à la complexité de la composition du produit final. II en résulte la production d'un médicament qui est hétérogène et comprend souvent de subtiles variantes moléculaires (microhétérogénéité) $(\rightarrow)$. A priori, le contrôle de la microhétérogénéité pourrait per- $\rightarrow$ voir L. Manache et al., page 1063 mettre d'identifier sur la base du profil spécifique de chaque produit, ceux qui sont contrefaits, voire ceux qui sont obtenus par le même process mais sur des sites de production différents. Cela pourrait aussi être un moyen de mesurer la variabilité qualitative des lots d'un produit sous licence.
L'enregistrement de biosimilaires est une réalité depuis la publication en 2006 des avis positifs du CHMP de I'EMEA pour deux demandes d'AMM pour des médicaments biosimilaires qui contiennent de la somatropine (hormone de croissance), Omnitrope et Valtropin. Néanmoins il reste à démonter que le développement d'Acm biosimilaires est réalisable, compte tenu de la complexité de ces molécules, et des conséquences sur la sécurité ou l'efficacité de modifications même mineures de la structure (microhétérogénéité) ou de la présence de traces d'impuretés. En conséquence de quoi la caractérisation physicochimique et biologique au cours du développement pourrait ne pas suffire et des données cliniques pourraient être, dans une certaine mesure, nécessaires pour confirmer la similitude clinique. Ceci est d'une importance clé pour les Acm et est clairement pris en compte par le cadre réglementaire européen. Des exigences réglementaires plus sévères pourraient encourager l'industrie pharmaceutique à développer des Acm contre de nouvelles cibles et pour de nouvelles applications, ce qui reste nécessaire pour améliorer le traitement de maladies rares.

\section{Perspectives d'avenir}

La classe des Acm devient de plus en plus connue et importante [7]. À titre d'exemple, Rituximab (MabThéra), un anticorps chimérique dirigé contre l'antigène CD20 sur les lymphocytes B a été, en 1997, le premier Acm autorisé par la FDA (Food and drug administration) pour le traitement d'hémopathies lymphoprolifératives. En effet, en dépit d'effets secondaires inévitables causés par un syndrome de libération des cytokines dû à la lyse massive des cellules cancéreuses, le Rituximab a démontré clairement son efficacité par rapport à la chimiothérapie classique si l'on prend comme critère l'amélioration de la survie à 3 ans des patients traités ([8], et $\rightarrow$ ). II fait maintenant partie du schéma thérapeutique stan- $(\rightarrow)$ voir G. Cartron et dard pour le traitement du lym- J.F. Rossi, page 1085 phome non hodgkinien, où il est administré sous forme d'association Rituximab-CHOP (cyclophosphamide, doxorubicine, vincristine et prednisolone) (R-CHOP). D'autres Acm sont déjà enregistrés ou en cours d'essais cliniques dans différentes applications: maladies auto-immunes, cardio-vasculaires, infectieuses, ophtalmologiques, dermatologiques, allergiques et rhumatologiques. Quelques Acm ont été autorisés pour le diagnostic in vivo.

Le nombre d'applications cliniques des Acm devrait augmenter dans l'avenir. Il est en particulier souhaitable 
que des Acm pour le traitement de maladies rares soient également développés, et que l'efficacité des traitements anticancéreux soit améliorée. En raison de leur origine murine, les Acm de première génération murins et chimériques seront progressivement remplacés par des anticorps humanisés et complètement humains, présentant moins d'effets secondaires $(\rightarrow)$. Par ailleurs, des Acm bio- $\quad(\rightarrow)$ voir M. Cogné et similaires produits à faible coût dans certains pays

al., page 1149 vont probablement apparaître bientôt sur le mar-

ché. L'aspect positif pour les patients et les systèmes de soins de santé, dont les budgets sont de plus en plus contraints, serait de réduire les coûts. Toutefois, le défi auquel les autorités de santé publique et les organismes de contrôle réglementaire auront à faire face est celui de l'élaboration de procédures nécessaires au maintien de normes de qualité élevées tout en assurant l'approvisionnement du marché par des médicaments de type Acm, dont le coût reste abordable pour nos sociétés. $\diamond$

\section{SUMMARY}

Regulatory consequences for the use of monoclonal antibodies

Köhler and Milstein published a method for the manufacture of mouse monoclonal antibodies of predefined specificity 1975 [1], a work rewarded with the Nobel Prize 1984. Since then, the field has developed rapidly with new production methods such as recombinant DNA technology, phage display and genetically engineered animals. Following the first clinical applications with a mouse monoclonal antibody, new classes as chimaeric, humanized and human monoclonal antibodies appeared, with the advantages of less adverse reactions and better efficacy. The development over more than 30 years resulted in more than 25 approved products on the market for various therapeutic applications, e.g. for the treatment of cancer, inflammatory diseases, heart disease and transplantation, and medicines for many more applications are currently under development. $\diamond$

\section{REMERCIEMENTS}

Les auteurs remercient Siegfried Giess (Paul-Ehrlich Institut, Allemagne) et Robin Thorpe (National Institute for Biological Standards and Control, Royaume-Uni) pour la lecture du manuscrit et de précieuses contributions et discussions.

\section{CONFLIT D'INTÉRÊTS}

Les auteurs déclarent n'avoir aucun conflit d'intérêts concernant les données publiées dans cet article.

\section{RÉFÉRENCES}

1. Köhler G, Milstein C. Continuous cultures of fused cells secreting antibody of predefined specificity. Nature 1975 ; 256 : 495-7.

2. Anticorps monoclonaux pour usage humain, monographie 2031. Pharmeuropa $2009 ; 21$ : 104-8.

3. Stebbings R, Findlay L, Edwards C, et al. "Cytokine storm" in the phase 1 trial of monoclonal antibody TGN1412 - better understanding the causes to improve pre-clinical testing of immunotherapeutics. J Immunol 2007 ; $179: 3325-31$.

4. Longstaff C. Personal communication.

5. Schneider CK, Kalinke U. Toward biosimilar monoclonal antibodies Nat Biotechnol $2008 ; 26: 985-90$.

6. Beck A, Lyer H, Reichert JM. European Medicines Agency workshop on biosimilar monoclonal antibodies Meeting Report (London, UK, July 2, 2009). mAbs-Landes Bioscience 2009; 1 : 394-416. (http://www. landesbioscience.com/journals/mabs/article/9630).

7. Reichert JM, Rosensweig CJ, Faden LB, Dewitz MC. Monoclonal antibody successes in the clinic. Nat Biotechnol $2005 ; 23$ : 1073-8.

8. McLaughlin P, Grillo-López AJ, Link BK, et al. Rituximab chimeric anti-CD20 monoclonal antibody therapy for relapsed indolent lymphoma: half of patients respond to a four-dose treatment program. J Clin Oncol 1998 ; $16: 2825-33$.

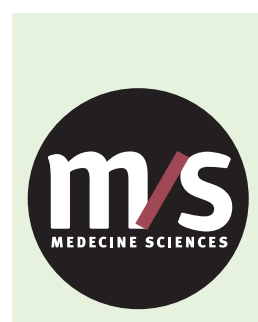

Tarifs d'abonnement M/S - 2010

Abonnez-vous

à Médecine/Sciences
$>$ Grâce à $m / s$, vous vivez en direct

les progrès des sciences biologiques et médicales

TIRÉS À PART

F. Lackner

\section{Bulletin d'abonnement page VI dans ce numéro de $\mathrm{m} / \mathrm{s}$}
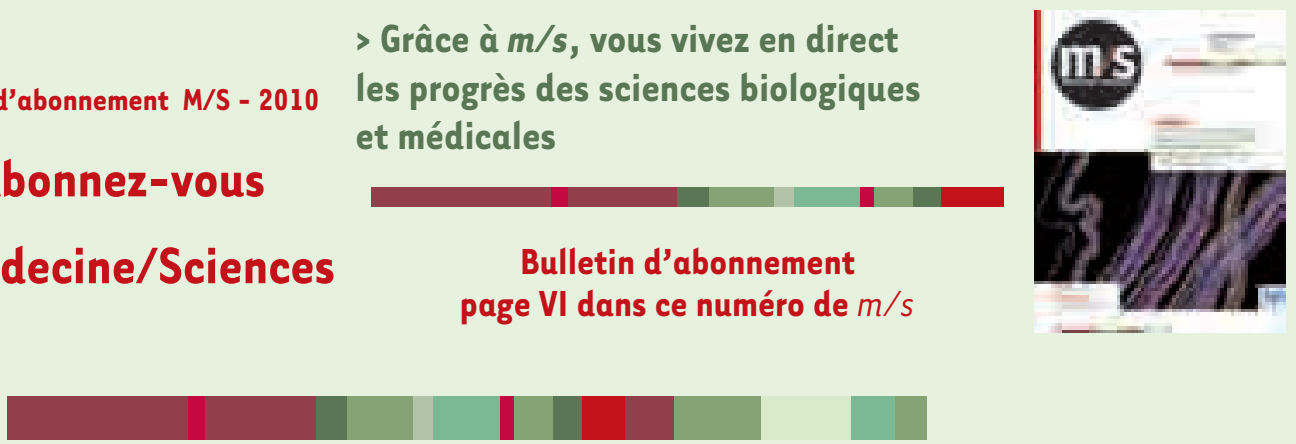

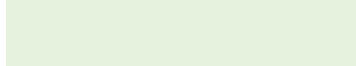

